\title{
Knowledge of Malaria and Antimalarial Drug Dispensing Practices in Buea Community
}

\author{
Marcelus U. Ajonina1, 2 \\ ${ }^{1}$ Health Sciences, Meridian Global University, Buea, CENTRAL REGION, Cameroon, ${ }^{2}$ Meridian Global Education and Research Foundation, Buea, \\ Southwest Region, Cameroon \\ Objective
}

This study was aimed at assessing the knowledge of malaria as well as perception and dispensing practices of antimalarials among vendors in Buea community

Introduction

Lack of knowledge of rational use of antimalarial drugs among dispensers is a serious problem, especially in areas of intense transmission thus increasing the risk of resistance and adverse drug reactions.

\section{Methods}

A community-based cross-sectional survey of a random sample of 140 drug vendors living within the Buea community was conducted between March and June 2017. Questionnaire was designed to obtain information from drug vendors on the general knowledge of malaria as well as dispensing practices. Data were analyzed using SPSS Statistics 20.0 and were considered significant at $\mathrm{P} \leq 0.05$

\section{Results}

Knowledge of malaria symptoms, transmission, and prevention was reasonable among $55.8 \%$ (77) of the respondents. Only $33.6 \%$ (47) of the respondents could attribute the cause of malaria to protozoan of genus Plasmodium species. Of the 140 vendors, $115(82.7 \%)$ prescribe antimalarial drugs. The knowledge of the national protocol was malaria case management among dispensers was $35.0 \%$. Vendors in hospital/community pharmacies were 2.4 times $(\mathrm{OR}=3.14,95 \% \mathrm{CI}$ :

4.14 - 8.74, $\mathrm{P}<0.001)$. more knowledgeable about malaria treatment protocol than those of in drugstores. The prevalence of selfprescription of antimalarials was $39.3 \%$. Self-prescription was significantly higher in drugstores than hospital/community pharmacies $(\mathrm{P}=0.004)$. In all, 56(40.6\%) of vendors showed good practices regarding antimalarial drug dispensing with majority $(51.7 \%)$ from community pharmacies $(\mathrm{OR}=2.27,95 \% \mathrm{CI}: 1.13-4.56)$.

\section{Conclusions}

Findings reveal moderate knowledge of malaria but poor prescription and dispensing practices of antimalarial drugs among vendors, thus indicating a need for routine monitoring and evaluation to prevent emergence of resistant strains to current efficacious antimalarials

\section{Acknowledgement}

We thank the all participants from various drug retail outlets from the Buea community who made this study possible by giving their consent. We equally thank Meridian Global Education and Research Foundation (MGERF), Cameroon for financial support. 